\title{
ISMGF 1987 World Stoke Mandeville Wheelchair Games
}

These annual World Games took place at the Ludwig Guttmann Sports Centre for the Disabled in Aylesbury, England, from 26 July to 2 August 1987.

They were the biggest games ever held here, apart from the Paralympics 1984, with an entry of some 720 athletes from 37 countries. Entering for the first time were teams from Nigeria, Puerto Rico and Sultanate of Oman. In addition, there were observers from Argentina, Chile and Singapore.

A new sport introduced into the Games was wheelchair tennis, which proved extremely popular. There was also an exhibition match between teams from Canada and Great Britain of Quadball-a team game for tetraplegics, which it is hoped will gain interest amongst other countries.

70 world records were broken-an indication of the increased standards and auguring well for the 1988 Seoul Paralympics.

The final day began with the Wheelchair Marathon, with an entry of 70 athletes. It was an Open Marathon and included the ISMGF event. Overall winner with a time of $1: 56: 43$ was Mike Truhillo of USA, which was all the more creditable in view of the very wet condition of the route, amounting to flooding in places.

The final of the basketball resulted in a win for the USA.

The rain cleared in time for the Closing Ceremony, which was performed by HRH The Prince of Wales, Royal Patron of the BPSS to the accompaniment of the lowering of flags, including that of the International Olympic Committee (IOC), which the latter had allowed us to fly throughout the Games.

Guests of Honour included Mr Kwi-Nam KOH, President of the 1988 Seoul Paralympic Organising Committee. It was also a great pleasure to welcome the new Minister of Sport, Colin Moynihan, to the Games, when he paid a visit on the last Saturday to meet the British Team and see something of the sporting events in which, as a sportsman himself, he was greatly interested.

Twenty-four hour coverage was given by a voluntary nursing rota from Stoke Mandeville Hospital and physician coverage by doctors from Pinderfields and Stoke Mandeville Hospitals. Three more doctors became ISMGF qualified medical classifiers after the Games, adding to the already considerable list spread throughout the world.

This should be especially helpful for the smooth running of the medical classification procedure in Seoul, as it is hoped that many teams will be able to utilise the availability of these medical classifiers to classify their ISMGF athletes beforehand. 